\title{
Osteoporosis in patients taking selective serotonin reuptake inhibitors: a focus on fracture outcome
}

\author{
Olivier Bruyère $\cdot$ Jean-Yves Reginster
}

Received: 6 February 2014/ Accepted: 3 July 2014/Published online: 5 August 2014

(C) Springer Science+Business Media New York 2014

\begin{abstract}
Depression is one of the most important mental health problems and a leading cause of disability. Selective serotonin reuptake inhibitors (SSRIs) are considered as first-line therapy for the treatment of depressive symptoms among older adults because of their presumed favorable adverse effect profile. However, they could have deleterious effects on the bone. Evidence from longitudinal, crosssectional, and prospective cohort studies suggests that the use of antidepressants at therapeutic doses is associated with decreased bone mineral density and increased fracture risk. The association between SSRIs use and fracture risk could potentially differ depending on dose, exposure duration, time of exposure, age, or sex. However, the risk of fracture declined rapidly after discontinuation of use of SSRIs. The evidence now seems sufficient to consider adding SSRIs to the list of medications that contribute to osteoporosis. In practice, assessment of risk factor for osteoporosis or fractures could be made taking into account age, gender, duration, and severity of depression, length of SSRI treatments, and other concurrent risk factors.
\end{abstract}

Keywords Depression - Osteoporosis · Fracture · Antidepressants $\cdot$ Selective serotonin reuptake inhibitors

Depression is a major public health problem and a leading cause of disability. Indeed, depression is one of the most important mental health problems, especially in the elderly, and it is associated with a poor natural history, reduced quality of life, and increased utilization of medical health

O. Bruyère $(\varangle)$ · J.-Y. Reginster

Department of Public Health, Epidemiology and Health

Economics, University of Liège, CHU Sart-Tilman, Bât B23,

4000 Liège, Belgium

e-mail: olivier.bruyere@ulg.ac.be services and high mortality [1]. Epidemiological studies suggest that the prevalence of some form of depression is about $20-25 \%$ in the Western population, with substantially higher rates in women than in men [2, 3]. The increased prevalence of depressive disorders has multiplied the prescription of antidepressant treatments $[4,5]$. According to a study from the National Health and Nutrition Examination Survey (NHANES), about half of patients with moderate or severe depressive symptoms have some form of treatment [2]. However, this figure reflects symptoms in a population cohort, many of whom will not have sought medical help or have a formal diagnosis of depression [6].

Antidepressants are one of the most commonly prescribed drugs in the Western world. Tricyclic antidepressants (TCAs) and selective serotonin reuptake inhibitors (SSRIs) are two of the most widely prescribed classes of antidepressants. However, SSRIs are considered as first-line therapy for the treatment of depressive symptoms among older adults because of their presumed favorable adverse effect profile [7]. The mechanisms of action of all these agents involve some impact on the serotonin system, though the degree of inhibition of the serotonin transporter (5-HTT) system may differ between classes. The SSRIs inhibit serotonin reuptake by the presynaptic neuron, thereby maintaining higher levels of serotonin in the synapse and increasing postsynaptic neurotransmission [8]. The serotonin molecule has some remarkable properties as recently reviewed [9]. It is synthesized by two different genes at two different sites and plays antagonistic functions on bone mass accrual at these two sites [9]. When produced peripherally, serotonin acts as a hormone to inhibit bone formation. In contrast, when produced in the brain, serotonin acts as a neurotransmitter to exert a positive and dominant effect on bone mass accrual by enhancing bone formation and limiting bone resorption. Anyway, most of the experimental in vitro 
and epidemiological studies have shown the negative impact of SSRI on bone.

Evidence from longitudinal, cross-sectional, and prospective cohort studies suggests that the use of antidepressants at therapeutic doses is associated with decreased bone mineral density (BMD) and increased fall and fracture risk [8, 10-19]. These associations have been demonstrated in several distinct populations, using various study designs and with bone density, bone loss, or fractures as outcomes [20]. Interestingly, these results are consistent after adjustment for confounding variables such as age, body mass index, lifestyle factors (alcohol, tobacco use), and history of fractures. It should be noted that alterations observed in BMD with the use of antidepressant drugs may take long to be of clinical importance and that the effects of short duration of use may have more clinical consequences on falls and the related fracture. However, new insights in the molecular pathways of bone remodeling have clarified the mechanisms responsible for skeletal fragility in several forms of secondary osteoporosis [21], and new methods have been proposed to investigate the bone quality in this clinical context [22].

Recently, some meta-analyses have been performed to review and quantitatively assess the effects of antidepressants medications and the risk of fracture [23-26]. Two of them have discussed specifically the effect of SSRIs [4, 24]. In the first one, published in 2012, Eom and Collaborators have included seven case-control and five cohort studies in the final analysis [25]. They have showed that the overall risk of fracture was higher among people using SSRIs (adjusted odds ratio [OR] 1.69, $95 \%$ confidence interval [CI] 1.51-1.90). The most recent meta-analysis, published in 2013 by our group, has included 16 studies [24]. It was shown that use of SSRI was associated with a $61,65,64$, and $22 \%$ of risk increase in fractures of all types, non-vertebral, hip, and spine fractures, respectively ([For fractures at any site: RR 1.61, $95 \%$ CI 1.49-1.74]; [For non-vertebral fractures: RR 1.65, $95 \%$ CI 1.44-1.89]; [For hip fractures: RR 1.64, $95 \%$ CI 1.42-1.89]; [For spine fractures: RR 1.22, $95 \%$ CI 1.05-1.42]). Interestingly, in this last meta-analysis, additional data highlighted that SSRI use showed systematically a higher increase in the risk of fractures of all types, non-vertebral, and hip fractures than studies evaluating TCA use.

All epidemiological studies assessing the effect of SSRIs on fractures are limited by the confounding variables included. Unfortunately, the types of confounders varied among the trials, and it seems that no consensus exists in the covariates used in risk adjustment. Anyway, in the meta-analysis of 2012, grouping studies by the number of adjustment variables for osteoporotic fracture risk factors revealed a higher increased fracture risk in those adjusting for fewer than four variables (adjusted OR 1.83,
$95 \%$ CI 1.57-2.13) than in those adjusting for four or more variables (adjusted OR 1.38, $95 \%$ CI 1.27-1.49) [25]. One of the important confounding variables is the depression status. Indeed, confounding by indication may be an important cause of the observed association since the medications are often prescribed for depressive symptoms, which have been associated, but not consistently, with lower bone mineral density, increased risk of falls, and increased risk of fractures in some studies [15, 27]. Moreover, it has been shown that antidepressant use is associated with poorer health status, poorer physical functioning, and other factors which are associated with an increased risk of fracture [8]. Interestingly, in our last meta-analysis, a less pronounced increase in fracture risk was observed in studies with adjustment for depression [14].

Hill criteria of causation are important to have in mind when considering causal relationship between a specific factor (e.g., SSRIs use) and a disease (e.g., fracture) [28]. Besides the strength of the association, the consistency of findings, the biological plausibility, the specificity of the association, and the temporal sequence of the association that have been discussed above, biological gradient (e.g., the dose-response) is also important when exploring the relationship between SSRIs and fracture. Interestingly, the association between the use of SSRIs and fracture differs depending on dose, exposure duration, time of exposure, age, or sex. Most of these data have been taken into account in the meta-analysis of Eom et al. [25]. The authors have shown that subgroup analysis by exposure duration revealed that the strength of association decreased with a longer window of SSRI administration before the index date. Same results were also nicely shown in a Dutch cohort where the risk of hip/femur fracture was increased during the first few months of continuous use of SSRIs, peaking at about 8 months, and remained elevated after about 1.5 years of continuous use [29]. The meta-analysis also showed that age, dose, and sex do not seem to have an impact on the strength of the association between SSRIs use and risk of fracture [25]. More recent data, however, seem to differ. Indeed, the large Danish case-control study of Vestergaard et al. [30], published in 2013, shows that for SSRI, a growing excess risk of fractures is seen with both increasing dose and age. More recently, in a large Canadian 10-year study, it was shown that participants taking higher doses of SSRI/SNRI at baseline had a significantly higher risk of fracture [19].

The risk of fracture after the end of use of antidepressant medications is also of primary importance. In this regards, one study is of particular interest. In this Dutch case-control study, van den Brand et al. have confirmed that the risk of hip/femur fracture increased with current use of SSRIs (adjusted OR 2.35, 95 \% CI 1.94-2.84]) [29]. However, 
they also showed a clear association between the time since the last intake of an SSRI and the risk of hip/femur fracture. The risk of hip/femur fracture, which was increased in current users, declined rapidly after discontinuation of use.

A few possible underlying mechanisms support the biological plausibility of these observations [15]. One explanation is that increased fracture risk is mediated simply by falling. Another explanation could involve the influence of serotonin on bone. SSRIs may thus, besides the effects related to falls, share effects on bone turnover and bone mineral density. Anyway, the evidence now seems sufficient to consider adding SSRIs to the list of medications that contribute to osteoporosis [8]. This would imply that clinicians consider osteoporosis management (i.e., bone mineral density assessment) for people taking SSRIs, or those taking SSRIs with some additional risk factors, for their risk of fracture. Interestingly, antidepressant use is not listed as a secondary cause of osteoporosis in the FRAX algorithm. Since the association between SSRIs use and fracture risk seems to be independent of bone mineral density, it may be useful to consider the possibility to have it included in FRAX. In practice, recommendations could be made at an individual level, taking into account age, gender, duration, and severity of depression, length of SSRI treatments, and other concurrent risk factors. At this stage, it seems appropriate to expect that providers of SSRIs have at least some discussion about bone health with their patients, with the final objective, from a public health point of view, to promote a healthy ageing [31].

\section{References}

1. E.S. Paykel, Depression: major problem for public health. Epidemiol. Psichiatr. Soc. 15(1), 4-10 (2006)

2. R.S. Shim, P. Baltrus, J. Ye, G. Rust, Prevalence, treatment, and control of depressive symptoms in the United States: results from the National Health and Nutrition Examination Survey (NHANES), 2005-2008. J. Am. Board Fam. Med. 24(1), 33-38 (2011). doi:10.3122/jabfm.2011.01.100121

3. J. Alonso, M.C. Angermeyer, S. Bernert, R. Bruffaerts, T.S. Brugha, H. Bryson, G. de Girolamo, R. Graaf, K. Demyttenaere, I. Gasquet, J.M. Haro, S.J. Katz, R.C. Kessler, V. Kovess, J.P. Lepine, J. Ormel, G. Polidori, L.J. Russo, G. Vilagut, J. Almansa, S. Arbabzadeh-Bouchez, J. Autonell, M. Bernal, M.A. BuistBouwman, M. Codony, A. Domingo-Salvany, M. Ferrer, S.S. Joo, M. Martinez-Alonso, H. Matschinger, F. Mazzi, Z. Morgan, P. Morosini, C. Palacin, B. Romera, N. Taub, W.A. Vollebergh, Prevalence of mental disorders in Europe: results from the European Study of the Epidemiology of Mental Disorders (ESEMeD) project. Acta Psychiatr. Scand. 190(s420), 21-27 (2004). doi:10.1111/j.1600-0047.2004.00327.x

4. M. Moore, H.M. Yuen, N. Dunn, M.A. Mullee, J. Maskell, T. Kendrick, Explaining the rise in antidepressant prescribing: a descriptive study using the general practice research database. BMJ 339, b3999 (2009). doi:10.1136/bmj.b3999

5. P. Lockhart, B. Guthrie, Trends in primary care antidepressant prescribing 1995-2007: a longitudinal population database analysis. Br. J. Gen. Pract. 61(590), e565-e572 (2011). doi:10. 3399/bjgp11X593848

6. R. Rizzoli, C. Cooper, J.Y. Reginster, B. Abrahamsen, J.D. Adachi, M.L. Brandi, O. Bruyere, J. Compston, P. Ducy, S. Ferrari, N.C. Harvey, J.A. Kanis, G. Karsenty, A. Laslop, V. Rabenda, P. Vestergaard, Antidepressant medications and osteoporosis. Bone 51(3), 606-613 (2012). doi:10.1016/j.bone.2012.05.018

7. O. Spigset, B. Martensson, Fortnightly review: drug treatment of depression. BMJ 318(7192), 1188-1191 (1999)

8. E.M. Haney, S.J. Warden, M.M. Bliziotes, Effects of selective serotonin reuptake inhibitors on bone health in adults: time for recommendations about screening, prevention and management? Bone 46(1), 13-17 (2010). doi:10.1016/j.bone.2009.07.083

9. P. Ducy, G. Karsenty, The two faces of serotonin in bone biology. J. Cell Biol. 191(1), 7-13 (2010). doi:10.1083/jcb.201006123

10. E.M. Haney, S.J. Warden, Skeletal effects of serotonin (5hydroxytryptamine) transporter inhibition: evidence from clinical studies. J. Musculoskelet. Neuronal Interact. 8(2), 133-145 (2008)

11. S.J. Diem, T.L. Blackwell, K.L. Stone, K. Yaffe, E.M. Haney, M.M. Bliziotes, K.E. Ensrud, Use of antidepressants and rates of hip bone loss in older women: the study of osteoporotic fractures. Arch. Intern. Med. 167(12), 1240-1245 (2007). doi:10.1001/ archinte.167.12.1240

12. L.J. Williams, M.J. Henry, M. Berk, S. Dodd, F.N. Jacka, M.A. Kotowicz, G.C. Nicholson, J.A. Pasco, Selective serotonin reuptake inhibitor use and bone mineral density in women with a history of depression. Int. Clin. Psychopharmacol. 23(2), 84-87 (2008). doi:10.1097/YIC.0b013e3282f2b3bb

13. E.M. Haney, B.K. Chan, S.J. Diem, K.E. Ensrud, J.A. Cauley, E. Barrett-Connor, E. Orwoll, M.M. Bliziotes, Association of low bone mineral density with selective serotonin reuptake inhibitor use by older men. Arch. Intern. Med. 167(12), 1246-1251 (2007). doi:10.1001/archinte.167.12.1246

14. R. Ginzburg, E. Rosero, Risk of fractures with selective serotonin-reuptake inhibitors or tricyclic antidepressants. Ann. Pharmacother. 43(1), 98-103 (2009). doi:10.1345/aph.1L264

15. S. Schwan, P. Hallberg, SSRIs, bone mineral density, and risk of fractures-a review. Eur. Neuropsychopharmacol. 19(10), 683-692 (2009). doi:10.1016/j.euroneuro.2009.05.001

16. V. Rabenda, O. Bruyere, J.Y. Reginster, Risk of nonvertebral fractures among elderly postmenopausal women using antidepressants. Bone 51(4), 674-679 (2012). doi:10.1016/j.bone.2012.07.030

17. M.S. Bakken, A. Engeland, L.B. Engesaeter, A.H. Ranhoff, S. Hunskaar, S. Ruths, Increased risk of hip fracture among older people using antidepressant drugs: data from the Norwegian Prescription Database and the Norwegian Hip Fracture Registry. Age Ageing 42(4), 514-520 (2013). doi:10.1093/ageing/aft009

18. D. Prieto-Alhambra, H. Petri, J.S. Goldenberg, T.P. Khong, O.H. Klungel, N.J. Robinson, F. de Vries, Excess risk of hip fractures attributable to the use of antidepressants in five European countries and the USA. Osteoporos. Int. 25(3), 847-855 (2014). doi:10.1007/s00198-013-2612-2

19. C. Moura, S. Bernatsky, M. Abrahamowicz, A. Papaioannou, L. Bessette, J. Adachi, D. Goltzman, J. Prior, N. Kreiger, T. Towheed, W.D. Leslie, S. Kaiser, G. Ioannidis, L. Pickard, L.A. Fraser, E. Rahme, Antidepressant use and 10-year incident fracture risk: the population-based Canadian Multicentre Osteoporosis Study (CaMoS). Osteoporos. Int. 25(5), 1473-1481 (2014). doi:10.1007/s00198-014-2649-x

20. J.J. Gagne, A.R. Patrick, H. Mogun, D.H. Solomon, Antidepressants and fracture risk in older adults: a comparative safety analysis. Clin. Pharmacol. Ther. 89(6), 880-887 (2011). doi:10. 1038/clpt.2011.54

21. G. Mazziotti, J. Bilezikian, E. Canalis, D. Cocchi, A. Giustina, New understanding and treatments for osteoporosis. Endocrine 41(1), 58-69 (2012). doi:10.1007/s12020-011-9570-2 
22. J.F. Griffith, H.K. Genant, New advances in imaging osteoporosis and its complications. Endocrine 42(1), 39-51 (2012). doi:10. 1007/s12020-012-9691-2

23. Q. Wu, W. Qu, M.D. Crowell, J.G. Hentz, K.A. Frey, Tricyclic antidepressant use and risk of fractures: a meta-analysis of cohort and case-control studies. J. Bone Miner. Res. 28(4), 753-763 (2013). doi:10.1002/jbmr.1813

24. V. Rabenda, D. Nicolet, C. Beaudart, O. Bruyere, J.Y. Reginster, Relationship between use of antidepressants and risk of fractures: a meta-analysis. Osteoporos. Int. 24(1), 121-137 (2013). doi:10. 1007/s00198-012-2015-9

25. C.S. Eom, H.K. Lee, S. Ye, S.M. Park, K.H. Cho, Use of selective serotonin reuptake inhibitors and risk of fracture: a systematic review and meta-analysis. J. Bone Miner. Res 27(5), 1186-1195 (2012). doi:10.1002/jbmr.1554

26. L.H. Oderda, J.R. Young, C.V. Asche, G.A. Pepper, Psychotropic-related hip fractures: meta-analysis of first-generation and second-generation antidepressant and antipsychotic drugs. Ann. Pharmacother. 46(7-8), 917-928 (2012). doi:10.1345/aph.1Q589
27. J.Y. Reginster, R. Deroisy, I. Paul, M. Hansenne, M. Ansseau, Depressive vulnerability is not an independent risk factor for osteoporosis in postmenopausal women. Maturitas 33(2), 133-137 (1999)

28. C.V. Phillips, K.J. Goodman, The missed lessons of Sir Austin Bradford Hill. Epidemiol. Perspect. Innov. 1(1), 3 (2004). doi: 10. 1186/1742-5573-1-3

29. M.W. van den Brand, S. Pouwels, M.M. Samson, T.P. van Staa, B. Thio, C. Cooper, H.G. Leufkens, A.C. Egberts, H.J. Verhaar, F. de Vries, Use of anti-depressants and the risk of fracture of the hip or femur. Osteopor. Int. 20(10), 1705-1713 (2009). doi:10. 1007/s00198-009-0849-6

30. P. Vestergaard, D. Prieto-Alhambra, M.K. Javaid, C. Cooper, Fractures in users of antidepressants and anxiolytics and sedatives: effects of age and dose. Osteoporos. Int. 24(2), 671-680 (2013). doi:10.1007/s00198-012-2043-5

31. J.M. Robine, E. Cambois, W. Nusselder, B. Jeune, H.V. Oyen, C. Jagger, The joint action on healthy life years (JA: EHLEIS). Arch. Public Health 71(1), 2 (2013). doi:10.1186/0778-7367-71-2 